\title{
Neurologic toxicity associated with immune checkpoint inhibitors: a pharmacovigilance study
}

\author{
Douglas B. Johnson 1* (D, Ali Manouchehri', Alexandra M. Haugh', Henry T. Quach', Justin M. Balko', \\ Benedicte Lebrun-Vignes ${ }^{2}$, Andrew Mammen ${ }^{3}$, Javid J. Moslehi ${ }^{1}$ and Joe-Elie Salem ${ }^{1,2}$
}

\begin{abstract}
Background: Immune checkpoint inhibitors (ICI) produce durable antitumor responses but provoke autoimmune toxicities, including uncommon but potentially devastating neurologic toxicities. The clinical features, including the spectrum, timing, and outcomes, of ICl-induced neurologic toxicities are not well characterized.

Methods: We performed disproportionality analysis using Vigibase, the World Health Organization pharmacovigilance database, comparing neurologic adverse event (AE) reporting in patients receiving ICls vs. the full database. Neurologic AEs were classified by group queries using Medical Dictionary for Regulatory Activities, between database inception to September 28, 2018. Associations between ICls and neurologic AEs were assessed using reporting odds ratios (ROR) and information component (IC). IC compares observed and expected values to find associations between drugs and AEs using disproportionate Bayesian reporting; IC $C_{025}$ (lower end of the IC 95\% credibility interval) $>0$ is considered statistically significant.

Results: Among the full database, 18,518,994 AEs were reported, including 48,653 with ICls. ICls were associated with higher incidence of myasthenia gravis $(0.47 \%$ of ICI reports vs. $0.04 \%$ of the full database, ROR $16.5[95 \% \mathrm{Cl} 14$. 5-18.9]; IC 025 3.31), encephalitis (0.51\% vs. 0.05\%, ROR 10.4 [95\% Cl 9.2-11.8]; IC $\left.C_{025} 3.15\right)$, peripheral neuropathy (1. $16 \%$ vs. $0.67 \%, I C_{025}$ 0.68), and meningitis (0.15\% vs. 0.06\%, ROR 3.1 [95\% Cl 2.5-3.9]; I $C_{025}$ 1.01). Myasthenia gravis and encephalitis were associated with anti-PD-1 whereas other neurologic AEs were associated with anti-CTLA-4. Myasthenia gravis was characterized by high fatality rates ( $20 \%)$, early onset (median 29 days), and frequent concurrent myocarditis and myositis; whereas other neurologic AEs had lower fatality rates (6-12\%), later onset (median 61-80 days), and were non-overlapping.
\end{abstract}

Conclusions: ICls produce a spectrum of distinct classes of neurologic AEs that can cause significant morbidity and mortality and tend to occur early and with class-specific associations.

Keywords: Neurotoxicity, PD-1, CTLA-4, PD-L1, Neuropathy, Encephalitis, Myasthenia gravis, Guillain-Barre syndrome

\section{Introduction}

Immune checkpoint inhibitors (ICI) have transformed the treatment landscape of numerous cancers, producing durable responses in a substantial fraction of patients [1]. Approved agents target programmed death-1 receptor (PD-1, nivolumab, pembrolizumab, cemiplimab), its ligand (PD-L1; atezolizumab, avelumab, durvalumab),

\footnotetext{
* Correspondence: Douglas.b.johnson@vumc.org

${ }^{1}$ Department of Medicine, Vanderbilt University Medical Center, 777 PRB, 2220 Pierce Ave, Nashville, TN 37232, USA

Full list of author information is available at the end of the article
}

and cytotoxic T lymphocyte antigen-4 (CTLA-4; ipilimumab) [2]. Toxic effects from these agents are related to removing nodes of self-tolerance and unleashing autoimmune-like phenomenon [3, 4]. Although usually manageable with corticosteroid administration, clinically severe events leading to morbidity and even mortality may complicate treatment [5].

Neurologic toxicities have emerged as clinically relevant complications of ICI. Case series have reported limited numbers of autoimmune-like, inflammatory events including encephalitis, aseptic meningitis, myasthenia

(c) The Author(s). 2019 Open Access This article is distributed under the terms of the Creative Commons Attribution 4.0 International License (http://creativecommons.org/licenses/by/4.0/), which permits unrestricted use, distribution, and reproduction in any medium, provided you give appropriate credit to the original author(s) and the source, provide a link to the Creative Commons license, and indicate if changes were made. The Creative Commons Public Domain Dedication waiver (http://creativecommons.org/publicdomain/zero/1.0/) applies to the data made available in this article, unless otherwise stated. 
gravis, and Guillain-Barre Syndrome which may occur, in aggregate, in $1-5 \%$ of treated patients [6-11]. However, a systematic analysis of the timing, spectrum, clinical associations, and outcomes of these uncommon events has not been performed in a large number of patients. Further, it remains unclear whether ICI are associated with other, more common neurologic events (such as cerebrovascular accident, seizures, multiple sclerosis, dementia, etc.). Defining the severe toxicities of ICI remains a critical objective given the rapidly increasing use of these agents, and long-term survival experienced by responding patients. While pharmacovigilance data may lack detailed clinical information, using this approach may help rigorously identify drug-toxicity associations. Herein, we leverage a large pharmacovigilance database (Vigibase), which has been used to characterize other ICI-induced toxicities [5, 12-16], to further define the neurologic toxicities associated with ICI.

\section{Methods}

\section{Study design and data sources}

This observational, retrospective, pharmacovigilance study is a disproportionality analysis based on adverse drug reactions reported in VigiBase, the WHO database of global, deidentified individual case safety reports (ICSRs), which includes reports from more than 130 countries [17]. VigiBase is managed by the Uppsala Monitoring Centre (UMC; Uppsala, Sweden), and contains more than 18.5 million ICSRs submitted by national pharmacovigilance centers since 1967. These reports originate from healthcare professionals, patients, or pharmaceutical companies, and are generally notified post-marketing.

\section{Procedures}

This study included all neurologic toxicities classified by group queries according to the Medical Dictionary for Regulatory Activities (MedDRA; Additional file 1: Table S1), between inception in November 14, 1967, and September 28, 2018. We categorized neurologic entities in the MedDRA classification based on the underlying pathophysiology, due to the overlap between different neurologic symptoms. Neurologic irAEs assessed in the analysis was limited to those suspected to be caused by ICIs. Each report in VigiBase contains administrative data (date of reporting, country of origin, qualification of reporter), patient characteristics (age and sex), drug characteristics (indication for treatment [cancer type], administration start and end dates, dose and regimen, route of administration), and reactions or events (reported terms including MedDRA classification terms, onset date, end date, seriousness, final outcome). We included ICIs in this study, including antibodies targeting PD-1 (nivolumab and pembrolizumab), PD-L1 (atezolizumab, avelumab, durvalumab), and CTLA-4 (ipilimumab, tremelimumab). Severe adverse events were defined as life-threatening events or those causing death, hospitalization, persistent or clinically significant disability, congenital anomaly, birth defect, or other significant medically important condition.

\section{Statistical analysis}

VigiBase allows for case/non-case analysis (also known as disproportionality analysis), which we used to study if suspected drug-induced neurologic events were differentially reported with ICIs as compared to neurologic events reported in the entire database with all the suspected drug-induced adverse reactions. Disproportionality analysis was also used to assess neurologic toxicities with different ICI regimens: anti-CTLA-4 monotherapy versus anti-PD-1/ PD-L1 monotherapy versus combination ICI therapy (anti-CTLA-4 and anti-PD-1/PD-L1 combination therapy). Disproportionality analysis compares the proportion of selected specific adverse-drug-reaction reported for a single or a group of drugs (e.g. ICI) with the proportion of the same adverse-drug-reaction for a control group of drugs (e.g. full database). The denominator in these analyses is the overall adverse-drug-reactions reported for each group of drugs. If the proportion of an adverse-drug-reaction is greater in patients exposed to a group of drug (cases) than in patients not exposed to this drug (non-cases), this suggests an association between the specific drug and the reaction and is a potential signal for safety. Disproportionality can be either calculated by the information component (IC) or reporting odds-ratio (ROR) when using full database as comparator, and only ROR when using different drug regimen subgroups as comparators.

Calculation of the IC, using a Bayesian confidence propagation neural network, was specifically developed and validated by UMC as a flexible, automated indicator value for disproportionate reporting that compares observed and expected drug-AE associations to find new drug-AE signals with identification of probability difference from the background data (full database) [18]. Probabilistic reasoning in intelligent systems (information theory) has been proven effective to manage large data sets, is robust in handling incomplete data, and may be used with complex variables. Information theory tools are ideal for finding drug-AE combinations with other variables, which are highly associated compared to the generality of the stored data [18]. Several examples with IC have been first validated showing the power of the technique to find signals very early after drug approval (e.g. captopril \pm coughing) and to avoid false positives where a common drug and a common $\mathrm{AE}$ association occur in the database, only because the drug is widely used and the $\mathrm{AE}$ frequently reported (e.g. digoxin \pm acne; digoxin \pm rash) $[18,19]$. Using VigiBase, this approach has recently been proven effective to characterize the spectrum of cardiovascular immune related adverse events associated with ICIs [16]. 
The statistical formula is as follows to calculate IC,

$$
\begin{aligned}
& \mathrm{IC}=\log 2\left(\left(\mathrm{~N}_{\text {observed }}+0.5\right) /\left(\mathrm{N}_{\text {expected }}+0.5\right)\right) \\
& \text { where } \mathrm{N}_{\text {expected }}=\left(\mathrm{N}_{\text {drug }} * \mathrm{~N}_{\text {effect }}\right) / \mathrm{N}_{\text {total }}
\end{aligned}
$$

$\mathrm{N}_{\text {expected: }}$ the number of case reports expected for the drug-adverse effect combination.

$\mathrm{N}_{\text {observed: }}$ the actual number of case reports for the drug-adverse effect combination.

$\mathrm{N}_{\text {drug: }}$ the number of case reports for the drug, regardless of adverse effects.

$\mathrm{N}_{\text {effect }}$ : the number of case reports for the adverse effect, regardless of drug.

$\mathrm{N}_{\text {total }}$ : the total number of case reports in the database.

$\mathrm{IC}_{025}$ is the lower end of a $95 \%$ credibility interval for the Information Component. A positive $\mathrm{IC}_{025}$ value $(>0)$ is the traditional threshold used in statistical signal detection at $\mathrm{UMC}[18,19] . \mathrm{IC}_{025}$ have only been validated for comparison of a drug versus full database and cannot be used to compare disproportionate reporting within different ICI regimens.

Disproportionality for neurologic irAE reporting as a function of variable ICI regimen was estimated by calculating the ROR Chi-square (Graphpad Prism 7), described elsewhere and detailed in Additional file 1: Table S2 [20-22]. The lower end of ROR 95\% confidence interval $(\mathrm{CI}) \geq 1$ is the threshold used for significant statistical signal detection. Characteristics of cases were described in terms of means ( \pm standard deviation) or medians (with interquartile range) for quantitative variables, and in terms of effective and proportion for qualitative ones. Time to toxicity was compared between different toxicities using the logrank test. Categorical variables were compared between toxicities using chi-square testing.

\section{Results}

A total of 48,653 adverse events were reported with ICI drugs, from a total number of 18,518,994 ICSRs reported in the full VigiBase dataset. Since ICI reports began in 2008 , we also note that $14,627,365$ ICSRs have been reported in VigiBase from 2008 - September 28, 2018. The numbers of different neurologic toxicities associated with ICI and in the full database are shown in Tables 1 and 2.

We identified five broad categories of neurologic events associated with ICI treatment compared with reporting from the full database. ICIs were associated with higher reporting of neuromuscular junction dysfunction $(0.47 \%$ of reports with ICIs vs. $0.04 \%$ for the full database, ROR 16.5 [95\% CI 14.5-18.9]; $\mathrm{IC}_{025} 3.31$ ), non-infectious encephalitis and/or myelitis $(0.51 \%$ vs. 0.05\%, ROR 10.4 [95\% CI 9.2-11.8]; $\mathrm{IC}_{025} 3.15$ ), cerebral artery vasculitis $(0.07 \%$ vs. $0.01 \%$; ROR $10.6[95 \% \mathrm{CI}$
7.5-14.9]; $\left.\mathrm{IC}_{025} 2.71\right)$, peripheral neuropathy (1.16\% vs. $\left.0.67 \%, \mathrm{IC}_{025} 0.68\right)$, and non-infectious meningitis $(0.15 \%$ vs. $0.06 \%$, ROR 3.1 [ $95 \%$ CI $2.5-3.9$ ]; $\mathrm{IC}_{025} 1.01$ ). The increased reporting of peripheral neuropathy was in part driven by acute polyneuropathies, specifically GuillainBarre syndrome which comprised 22\% $(n=122 / 564)$ of peripheral neuropathy cases and were disproportionately associated with ICI $(0.25 \%$ vs. $0.05 \%$, ROR 4.7 [95\% CI 3.9-5.6], $\mathrm{IC}_{025} 2.00$ ). Notably, most cases of cerebral artery vasculitis reported were temporal arteritis $(n=23$, $67.7 \%)$, although cerebral vasculitis $(n=8)$ was also observed. Since we recently described temporal arteritis and other vasculitis syndromes associated with ICI, [16] we chose not to focus on these further; characteristics of patients with CNS vasculitis are listed in Additional file 1: Table S3. Other neurologic events, including hemorrhagic or ischemic strokes, seizures, headaches, extrapyramidal syndromes, dementia, sleep disturbances, psychotic disorders, or demyelinating disorders did not have increased reporting with ICIs (Table 1 ).

The associations of neurologic irAEs with class of ICI (anti-PD-1/PD-L1 vs. anti-CTLA-4 vs. combination) are listed in Table 2. Notably, we observed distinct events associated with different classes. Neuromuscular junction dysfunction (myasthenia gravis) was over-reported in patients treated with anti-PD-1/PD-L1 compared with anti-CTLA-4 (ROR 3.9, 95\% CI 2.3-6.8). Non-infectious encephalitis/myelitis was reported more with anti-PD-1/ PD-L1 than with anti-CTLA-4 (ROR 2.5, 95\% CI 1.6-3.9) and with combination therapy compared with monotherapy (ROR 2.0, 95\% CI 1.4-2.7). By contrast, Guillain-Barre syndrome (GBS) (ROR $0.48,95 \%$ CI $0.32-0.72$ ) and non-infectious meningitis (ROR 0.5 , 95\% CI $0.29-0.86$ ) were less frequently reported with anti-PD-1/PD-L1 compared with anti-CTLA4 monotherapy, and were more frequently associated with combination PD-1/PD-L1 + CTLA-4 blockade compared with monotherapy (ROR 2.0 [95\% CI 1.2-3.1] and ROR 2.7 [95\% CI 1.5-4.7] for GBS and meningitis, respectively). Similar OR were observed with individual anti-PD-1 vs. anti-PD-L1 drugs, although a low number of anti-PD-L1 associated events were noted (data not shown). IC values and their $95 \%$ credibility intervals over time are shown in Fig. 1 for neuromuscular junction disorders, encephalitis, GBS, and meningitis.

To assess the clinical features of these neurologic irAEs associated with ICIs, we described the clinical characteristics of patients who developed neuromuscular junction dysfunction $(n=228)$, noninfectious encephalitis/myelitis $(n=$ 250), Guillain-Barre Syndrome $(n=122)$, and noninfectious meningitis $(n=72)$ (Table 3). Most cases were reported in $2017-2018$ (61-78\% of cases), reflecting the substantially increased use of ICIs in recent years. Toxicities were substantially more common in men than women, ranging from 53 to $65 \%$ men depending on the toxicity. Encephalitis/ 
Table 1 Neurologic immune-related adverse events reported with ICls versus those reported in the full database from VigiBase, from Nov 14, 1967, to September 28, 2018

\begin{tabular}{|c|c|c|c|}
\hline & Overall ICls & Full database (starting 1967) & $\mathrm{IC} / \mathrm{IC}_{025}$ \\
\hline Total number of ICSRs available & 48,653 & $18,518,994$ & \\
\hline \multicolumn{4}{|l|}{ Number of ICSRs by irAE subgroups } \\
\hline Neuromuscular junction dysfunction (myasthenia gravis) & $228(0.47 \%)$ & $7455(0.04 \%)$ & $3.51 / 3.31$ \\
\hline Noninfectious encephalitis and/or myelitis & $250(0.51 \%)$ & $9267(0.05 \%)$ & $3.33 / 3.15$ \\
\hline Cerebral artery vasculitis & $34(0.07 \%)$ & $1206(0.01 \%)$ & $3.23 / 2.71$ \\
\hline Peripheral neuropathy & $564(1.16 \%)$ & $123,463(0.67 \%)$ & $0.80 / 0.68$ \\
\hline - Guillain-Barre syndrome & $122(0.25 \%)$ & $9508(0.05 \%)$ & $2.27 / 2.00$ \\
\hline - Chronic polyneuropathies & $23(0.05 \%)$ & $6428(0.03 \%)$ & $0.43 /-0.22$ \\
\hline - Mononeuropathies & $42(0.09 \%)$ & $17,075(0.09 \%)$ & $-0.09 /-0.58$ \\
\hline Noninfectious meningitis & $72(0.15 \%)$ & $10,532(0.06 \%)$ & $1.36 / 1.01$ \\
\hline Hemorrhagic central nervous system vascular conditions & $386(0.79 \%)$ & $195,577(1.06 \%)$ & $-0.41 /-0.56$ \\
\hline Cranial nerve disorders (excluding neoplasms) & $226(0.46 \%)$ & $112,639(0.61 \%)$ & $-0.39 /-0.58$ \\
\hline Cerebral ischemia & $332(0.68 \%)$ & $174,768(0.94 \%)$ & $-0.47 /-0.63$ \\
\hline Spinal cord and nerve root disorders & $27(0.06 \%)$ & $11,875(0.06 \%)$ & $-0.21 /-0.80$ \\
\hline Speech and language abnormalities & $215(0.44 \%)$ & $125,871(\%)$ & $-0.62 /-0.82$ \\
\hline Seizures & $291(0.60 \%)$ & $238,924(0.68 \%)$ & $-1.11 /-1.28$ \\
\hline Headaches & $776(1.59 \%)$ & $731,460(3.95 \%)$ & $-1.31 /-1.41$ \\
\hline Coma states & $56(0.12 \%)$ & $43,228(0.23 \%)$ & $-1.01 /-1.41$ \\
\hline Extrapyramidal syndrome & $854(1.76 \%)$ & $840,831(4.54 \%)$ & $-1.37 /-1.47$ \\
\hline Sensory abnormalities & $520(1.07 \%)$ & $551,559(2.98 \%)$ & $-1.48 /-1.60$ \\
\hline Dementia & $21(0.04 \%)$ & $15,706(0.08 \%)$ & $-0.96 /-1.64$ \\
\hline Movement disorders & $396(0.81 \%)$ & $427,006(2.31 \%)$ & $-1.50 /-1.65$ \\
\hline Vertigos & $89(0.18 \%)$ & $91,034(0.49 \%)$ & $-1.42 /-1.74$ \\
\hline Nervous system neoplasms benign & $5(0.01 \%)$ & $2302(0.01 \%)$ & $-0.25 /-1.78$ \\
\hline Sleep disturbances & $239(0.49 \%)$ & $314,528(1.70 \%)$ & $-1.79 /-1.98$ \\
\hline Psychosis and psychotic disorders & $183(0.38 \%)$ & $284,048(1.53 \%)$ & $-2.03 /-2.24$ \\
\hline Demyelinating disorders & $38(0.08 \%)$ & $87,190(0.47 \%)$ & $-2.58 /-3.07$ \\
\hline
\end{tabular}

Data are $N$ (\%) unless otherwise stated. ICls refers to any ICSRs reported for treatment with nivolumab, pembrolizumab, atezolizumab, avelumab, durvalumab, ipilimumab, or tremelimumab. A positive $\mathrm{IC}_{025}$ value $(>0)$ is the traditional threshold used in statistical signal detection with VigiBase. ICSRs individual case safety reports. ICls immune checkpoint inhibitors. IC information component. IC025 = lower end of a 95\% credibility interval for the IC

Bold text denotes statistically significant differences

myelitis and meningitis appeared to occur in slightly younger patients (median ages 58.6 and 56.3 years, respectively) compared with myasthenia gravis and GBS (median 70.3 and 65 years, respectively). Cases largely were reported in patients with lung cancer $(33 \%$ of all cases; $n=188 / 574)$ and melanoma (36\%; $n=206 / 574)$. Comparing lung cancer and melanoma, myasthenia gravis and encephalitis/myelitis were more common in lung cancer (163 vs. 103 cases) whereas GBS and meningitis were more common in melanoma (103 vs. 25 cases; $p<0.0001$ ) although this may reflect the differential use of causative therapies in these cancers (e.g. events associated with ipilimumab occurred more often in melanoma patients).

Neurologic events generally occurred within the first three months after starting ICI therapy; however myasthenia gravis (median 29 days) had more rapid onset than other events (median onset 61-80 days; $p<0.001$ ) (Fig. 2a). Myasthenia gravis also had the highest fatality rates $(44 / 228$, $19.3 \%)$ compared with other neurologic toxicities (51/444, $11.5 \% ; p=0.024)$. Myasthenia gravis presenting with both myocarditis and myositis had the highest death rate $(5 / 8$; $62.5 \%)$, compared with myasthenia gravis alone (29/179; $16.2 \%)$, or myasthenia gravis presenting with either myositis alone $(6 / 29 ; 20.7 \%)$, or with myocarditis alone (4/12; $33 \%)$. Among patients who died with available data, median time to death was 43 days (myasthenia gravis), 64.5 days (Guillain-Barre), 60.8 days (encephalitis), and 42 days (meningitis). Neurologic AEs were rarely overlapping, with the exception of encephalitis and meningitis in 5 patients (Fig. 2b). Most patients did not have concurrent severe non-neurologic 
Table 2 Selected neurological adverse events (detected as signals) reported for ICls versus the full database from VigiBase, from Jan 1, 2008, to September 28, 2018

\begin{tabular}{|c|c|c|c|c|c|c|c|}
\hline \multirow[t]{3}{*}{ Total number of ICSRs } & \multicolumn{3}{|c|}{ Overall ICls (n:) } & \multirow{3}{*}{$\begin{array}{l}\text { Full database } \\
\text { (full; starting 2008 } \\
\text { N:14,627,365) }\end{array}$} & \multirow{3}{*}{$\begin{array}{l}\text { ROR and 95\% } \\
\text { CI [,] anti-PD-1 } \\
\text { or anti-PD-L1 } \\
\text { vs anti-CTLA-4 } \\
\text { monotherapy }\end{array}$} & \multirow{3}{*}{$\begin{array}{l}\text { ROR and } \\
95 \% \mathrm{Cl}[\text { ]] } \\
\text { combination } \\
\text { ICls vs } \\
\text { monotherapy }\end{array}$} & \multirow{3}{*}{$\begin{array}{l}\text { ROR and 95\% } \\
\mathrm{Cl}[\mathrm{l}] \mathrm{ICls} \text { vs full } \\
\text { database }\end{array}$} \\
\hline & \multicolumn{2}{|c|}{ MONO (N:43,960) } & \multirow{2}{*}{$\begin{array}{l}\text { COMB } \\
(N: 4693)\end{array}$} & & & & \\
\hline & $\begin{array}{l}\text { MONO-PD1 } \\
(N: 34,401)\end{array}$ & $\begin{array}{l}\text { MONO-CTLA4 } \\
(N: 9559)\end{array}$ & & & & & \\
\hline \multicolumn{8}{|c|}{ Number of ICSRs by Neuro-ADR subgroup } \\
\hline $\begin{array}{l}\text { Neuromuscular junction } \\
\text { dysfunction }\end{array}$ & $197(0.57 \%)$ & $14(0.15 \%)$ & $17(0.36 \%)$ & $4380(0.03 \%)$ & $3.9[2.3-6.8]$ & $0.8[0.5-1.2]$ & 16.5 [14.5-18.9] \\
\hline $\begin{array}{l}\text { Non-infectious encephalitis } \\
\text { and/or myelitis }\end{array}$ & $186(0.54 \%)$ & $21(0.22 \%)$ & $43(0.92 \%)$ & $7460(0.05 \%)$ & $2.5[1.6-3.9]$ & $2[1.4-2.7]$ & $10.4[9.2-11.8]$ \\
\hline Guillain-Barre syndrome & $64(0.19 \%)$ & $37(0.39 \%)$ & $21(0.45 \%)$ & $7962(0.05 \%)$ & $0.48[0.32-0.72]$ & $2[1.2-3.1]$ & $4.7[3.9-5.6]$ \\
\hline Non-infectious meningitis & $36(0.10 \%)$ & $20(0.21 \%)$ & $16(0.34 \%)$ & $6986(0.04 \%)$ & $0.5[0.29-0.86]$ & $2.7[1.5-4.7]$ & $3.1[2.5-3.9]$ \\
\hline
\end{tabular}

Abbreviations: Mono monotherapy, COMB combination therapy, PD1 Programmed death-1/ligand-1, CTLA4 cytotoxic T lymphocyte antigen-4 Data are $N(\%)$ unless otherwise stated. ICls refers to any ICSR reported for treatment with nivolumab, pembrolizumab, atezolizumab, avelumab, durvalumab, ipilimumab, or tremelimumab. Anti-PD-1 or anti-PD-L1 monotherapy refers to any ICSR associated with any of the following five drugs only when used alone: nivolumab, pembrolizumab, atezolizumab, avelumab, or durvalumab. Anti-CTLA-4 monotherapy refers to any ICSR associated with ipilimumab or tremelimumab alone. Combination ICls refers to any ICSR reported with at least one anti-PD-1 or anti-PD-L1 drug combined with an anti-CTLA-4 drug. ICSRs $=$ individual case safety reports. $\mathrm{ICls}=$ immune checkpoint inhibitors. $\mathrm{ROR}=$ reporting odds ratio

${ }^{a}$ First reports of ICSRs associated with ICls started in 2008

Bold text denotes statistically significant differences

irAEs, with the exception of patients with myasthenia gravis, which often had myocarditis (8.8\%), myositis (16.2\%), or both. Myasthenia gravis $(74 / 228 ; 32.5 \%)$ and meningitis $(26 / 72 ; 36.1 \%)$ were more likely to have concurrent irAEs compared with other neurotoxicities (45/250, $18 \%$ and 12/122, $9.9 \%$ for encephalitis and GBS, respectively; $p<0.0001)$. Additional clinical descriptions are shown in Additional file 1: Tables S4-S7.

\section{Discussion}

This study reports, to our knowledge, the largest characterization of neurologic irAEs associated with ICIs
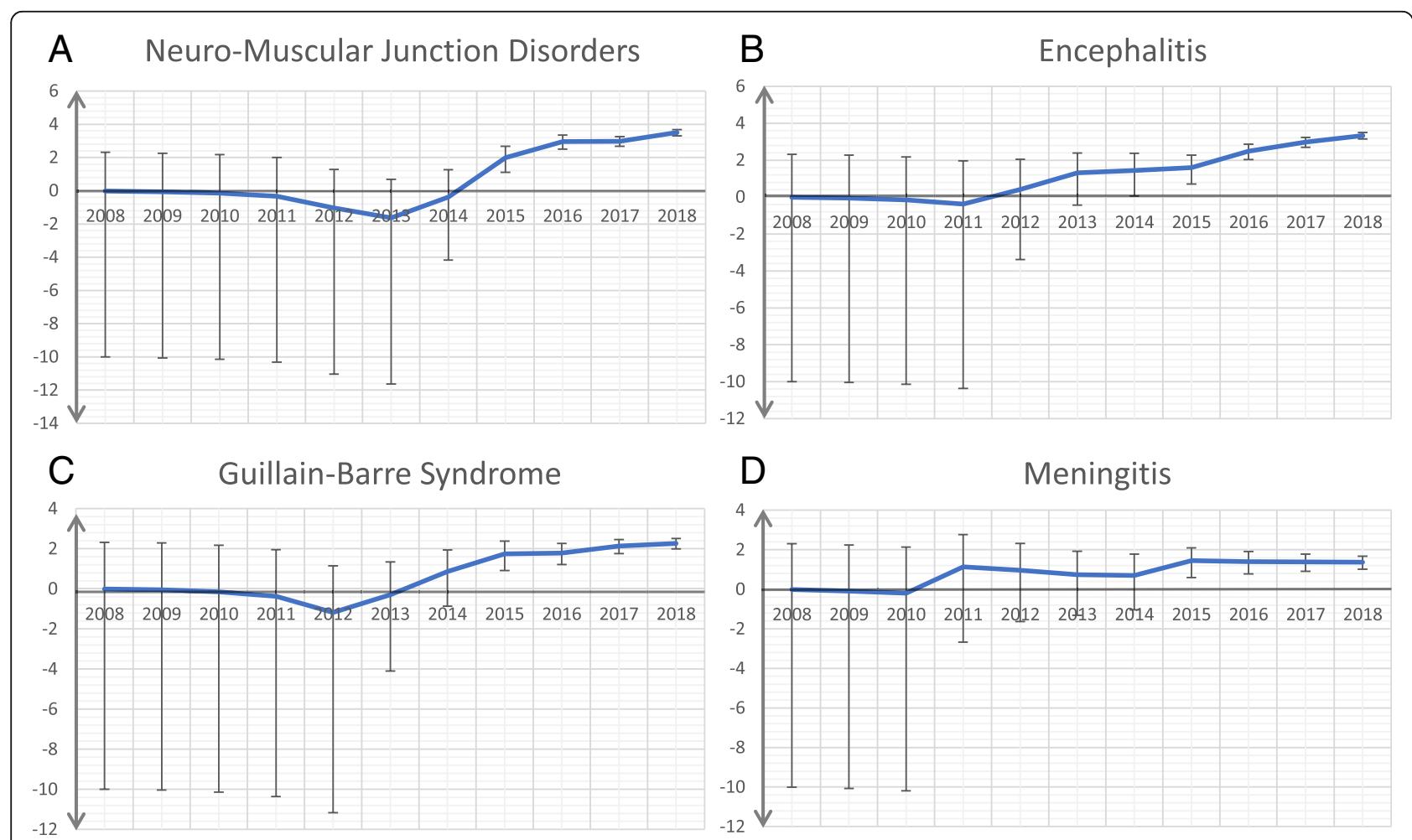

Fig. 1 Information component and its 95\% credibility interval over time for (a) neuromuscular junction disorders, (b) encephalitis/myelitis, (c) Guillain-Barre Syndrome, and (d) meningitis 
Table 3 : Clinical characteristics of patients with $\mathrm{ICl}$ induced neurotoxicities

\begin{tabular}{|c|c|c|c|c|}
\hline Characteristics & $\begin{array}{l}\text { Myasthenia gravis } \\
N(\%)^{\mathrm{a}}\end{array}$ & $\begin{array}{l}\text { Encephalitis/ myelitis } \\
N(\%)^{\mathrm{a}}\end{array}$ & $\begin{array}{l}\text { Guillain-Barre syndrome } \\
N(\%)^{\mathrm{a}}\end{array}$ & $\begin{array}{l}\text { Non-infectious meningitis } \\
N(\%)^{\mathrm{a}}\end{array}$ \\
\hline Total number & 228 & 250 & 122 & 72 \\
\hline \multicolumn{5}{|l|}{ Reporting year } \\
\hline 2018 (through September) & $101(44.30)$ & $107(42.80)$ & $45(36.9)$ & $20(27.78)$ \\
\hline 2017 & $73(32.02)$ & $90(36.00)$ & $46(37.7)$ & $24(33.33)$ \\
\hline 2016 & $39(17.11)$ & $38(15.20)$ & $17(13.9)$ & $13(18.06)$ \\
\hline 2015 & $12(5.26)$ & $8(3.20)$ & $7(5.7)$ & $9(12.50)$ \\
\hline 2014 & $3(1.32)$ & $3(1.20)$ & $6(4.9)$ & $3(4.17)$ \\
\hline 2012-2013 & 0 & $4(1.60)$ & $1(0.82)$ & $3(4.17)$ \\
\hline \multicolumn{5}{|l|}{ Gender } \\
\hline Male & $125(60.98)$ & $140(63.35)$ & $71(65.74)$ & $35(53.03)$ \\
\hline Female & 80 (39.02) & 81 (36.94) & 37 (34.26) & $31(46.97)$ \\
\hline Data available & $205(89.91)$ & $221(88.40)$ & $108(88.52)$ & $66(91.67)$ \\
\hline Age at onset, mean $\pm S D$, years & $70.28 \pm 10.50$ & $58.69 \pm 15.98$ & $65.01 \pm 14.41$ & $56.25 \pm 14.63$ \\
\hline [min-max] & [32-86] & {$[7-86]$} & $(25-98)$ & {$[28,86]$} \\
\hline Data available & $122(53.51)$ & $162(64.80)$ & $76(62.29)$ & $51(70.83)$ \\
\hline \multicolumn{5}{|l|}{ Drugs } \\
\hline Monotherapy with Anti PD-1/PD-L1 & $197(86.40)$ & $186(74.40)$ & $64(52.46)$ & $36(50.00)$ \\
\hline - Nivolumab & $68(34.52)$ & $127(68.28)$ & $33(51.56)$ & $23(63.89)$ \\
\hline - Pembrolizumab & $116(58.88)$ & $36(19.35)$ & $27(42.19)$ & $9(25.00)$ \\
\hline - Atezolizumab & $9(4.57)$ & $20(10.58)$ & $3(4.69)$ & $4(11.11)$ \\
\hline - Durvalumab & $1(0.51)$ & $3(1.59)$ & $0(0)$ & $0(0)$ \\
\hline - Avelumab & $3(1.52)$ & $0(0)$ & $1(0.82)$ & $0(0)$ \\
\hline Monotherapy with Anti CTLA-4 & $14(6.14)$ & $21(8.40)$ & $37(30.33)$ & $20(27.78)$ \\
\hline - Ipilimumab & $14(100)$ & $20(100)$ & $37(100)$ & $20(100)$ \\
\hline Combination therapy & $17(7.46)$ & $43(17.20)$ & $21(17.21)$ & $16(22.22)$ \\
\hline - Nivolumab + Ipilimumab & $15(88.24)$ & $40(93.02)$ & $20(95.24)$ & $16(100)$ \\
\hline - Pembrolizumab + Ipilimumab & $1(5.88)$ & $3(6.98)$ & $1(4.76)$ & $0(0)$ \\
\hline - Tremelimumab + Durvalumab & $1(5.88)$ & $0(0)$ & $0(0)$ & $0(0)$ \\
\hline \multicolumn{5}{|l|}{ Indications } \\
\hline Lung cancer & $73(39.67)$ & $90(38.96)$ & $18(18.75)$ & $7(11.11)$ \\
\hline Malignant melanoma & $47(25.54)$ & $56(24.24)$ & $65(67.71)$ & $38(60.32)$ \\
\hline Renal cell carcinoma & $25(13.59)$ & $13(5.62)$ & $5(5.21)$ & $5(7.94)$ \\
\hline Other & $39(21.20)$ & $51(20.40)$ & $8(8.33)$ & $13(20.63)$ \\
\hline Data available & $184(80.70)$ & $231(85.20)$ & $96(78.69)$ & $63(87.50)$ \\
\hline \multicolumn{5}{|l|}{ Time to irAE onset, days: } \\
\hline Median, [IQR] & 29 [24-53] & $61[18-153]$ & $65.5[29-124]$ & $68[27-134]$ \\
\hline$[\min -\max ]$ & [6-132] & [1-841] & [2-995] & [8-400] \\
\hline Data available & $45(19.74)$ & $72(28.80)$ & $34(27.87)$ & $23(31.51)$ \\
\hline Death & $44(19.30)$ & $32(12.80)$ & $13(10.66)$ & $6(8.33)$ \\
\hline \multicolumn{5}{|c|}{ Concurrent neurologic symptoms/syndromes } \\
\hline Myasthenia gravis & N/A & $1(0.40)$ & $1(0.82)$ & $0(0)$ \\
\hline Encephalitis/myelitis & $1(0.44)$ & N/A & $1(0.82)$ & $5(6.94)$ \\
\hline Cerebral vasculitis & $0(0)$ & $1(0.40)$ & $0(0)$ & $1(1.39)$ \\
\hline
\end{tabular}


Table 3 : Clinical characteristics of patients with ICl induced neurotoxicities (Continued)

\begin{tabular}{|c|c|c|c|c|}
\hline Characteristics & $\begin{array}{l}\text { Myasthenia gravis } \\
N(\%)^{\mathrm{a}}\end{array}$ & $\begin{array}{l}\text { Encephalitis/ myelitis } \\
N(\%)^{\mathrm{a}}\end{array}$ & $\begin{array}{l}\text { Guillain-Barre syndrome } \\
N(\%)^{\mathrm{a}}\end{array}$ & $\begin{array}{l}\text { Non-infectious meningitis } \\
N(\%)^{\mathrm{a}}\end{array}$ \\
\hline Guillain Barre syndrome & $1(0.44)$ & $1(0.40)$ & N/A & $2(2.78)$ \\
\hline Peripheral Neuropathy & $0(0)$ & $4(1.60)$ & N/A & $0(0)$ \\
\hline Meningitis & $0(0)$ & $5(5.00)$ & $2(1.64)$ & N/A \\
\hline Demyelination & $0(0)$ & $5(2.00)$ & $0(0)$ & $0(0)$ \\
\hline Seizure & $1(0.44)$ & $10(4.00)$ & $0(0)$ & $0(0)$ \\
\hline Stroke & $0(0)$ & $2(0.80)$ & $0(0)$ & $4(5.56)$ \\
\hline Blindness (unilateral or bilateral) & $1(0.44)$ & $0(0)$ & $0(0)$ & $0(0)$ \\
\hline Coma/loss of consciousness & $1(0.44)$ & $4(1.60)$ & $0(0)$ & $0(0)$ \\
\hline \multicolumn{5}{|l|}{ Other irAEs } \\
\hline Colitis/diarrhea & $4(1.75)$ & $4(1.60)$ & $3(2.46)$ & $3(4.17)$ \\
\hline Pneumonitis & $1(0.44)$ & $5(2.00)$ & $0(0)$ & $2(2.78)$ \\
\hline Myocarditis & $20(10.52)$ & $3(1.20)$ & $0(0)$ & $1(1.39)$ \\
\hline Myositis & $37(16.23)$ & $2(0.80)$ & $0(0)$ & $1(1.39)$ \\
\hline Dermatitis & $2(0.88)$ & $12(4.80)$ & $3(2.46)$ & $3(4.17)$ \\
\hline Thyroiditis/hypothyroidism & $7(3.07)$ & $6(2.40)$ & $5(4.10)$ & $1(1.39)$ \\
\hline Hypophysitis/hypopituitarism & $0(0)$ & $5(2.00)$ & $1(0.82)$ & $5(6.94)$ \\
\hline Hepatitis & $11(4.82)$ & $2(0.80)$ & $3(2.46)$ & $1(1.39)$ \\
\hline Nephritis & $0(0)$ & $2(0.80)$ & $0(0)$ & $1(1.39)$ \\
\hline Other & $3(1.32)$ & $10(4.00)$ & $2(1.64)$ & $7(9.72)$ \\
\hline None & $154(67.54)$ & $205(82.00)$ & $110(90.16)$ & $46(63.89)$ \\
\hline
\end{tabular}

${ }^{\mathrm{a}}$ Data available $=100 \%$ unless noted

Abbreviations: CTLA-4 cytotoxic T-lymphocyte-associated protein $4, I C l$ immune checkpoint inhibitor, $I Q R$ interquartile range, irAE immune related adverse event, [min-max] minimum-maximum, PD-1 programmed cell death protein 1, PD-L1 programmed cell death ligand 1, SD standard deviation, N/A not applicable

by detailed analysis of a global, WHO database (VigiBase). ICIs were significantly associated with over reporting of five distinct categories of neurologic toxicities: neuromuscular dysfunction (myasthenia gravis), encephalitis/myelitis, cerebral vasculitis, Guillain-Barre Syndrome, and non-infectious meningitis. While the clinical features of a limited number of these cases have been reported previously, this is the first effort to systematically associate their occurrence with ICI use, and characterize a large population of affected patients.

ICIs function by removing key regulators of immune tolerance; thus toxicities affecting other organ systems have largely been related to autoimmune-type and inflammatory phenomenon. The events observed in our
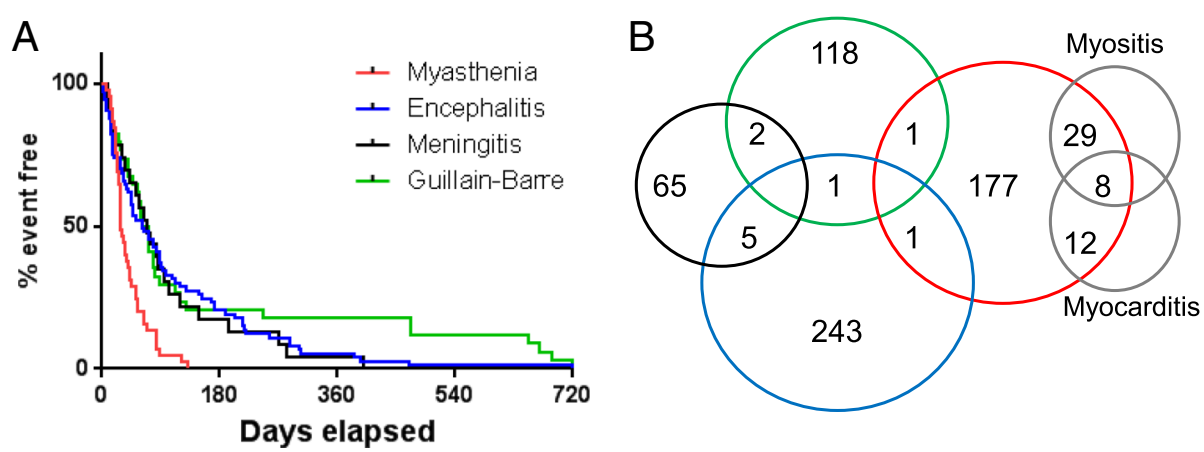

Fig. 2 a Time to event for different categories of neurotoxicities. b Modified Venn diagram showing overlap between distinct classes of neurotoxicities (color scheme the same as in A), as well myositis and myocarditis. Blank cells have no cases for neurotoxicities (e.g. no reports of concurrent myasthenia gravis, GBS, and encephalitis) or were beyond the scope of this study (myositis and myocarditis cases without overlapping neurotoxicities) 
study largely recapitulate these findings, with inflammatory manifestations (encephalitis, vasculitis, meningitis), and/or autoimmune syndromes (myasthenia gravis and GBS). Interestingly, these events were differentially associated either with single-agent anti-PD-1/PD-L1 (myasthenia gravis and encephalitis) or anti-CTLA-4 (GBS and meningitis), and were generally increased by combination ICI therapy. Neurotoxicities tended to occur fairly early on therapy (median onset within 3 months for all categories), although only myasthenia gravis had a hyperacute onset (similar to myocarditis or fatal irAEs) $[5,12]$. The severity of these events varied, with fatality rates similar to irAEs involving other organ systems (approximately $10 \%$ for most events) [5]. Notably, the low number of events observed (concordant with lower use overall) of anti-PD-L1 drugs (vs. anti-PD-1) highlights the need to study differences between these classes.

Myasthenia gravis appeared to have the most fulminant presentation, with a nearly $20 \%$ fatality rate, early onset (median 29 days), and frequent association with myositis and myocarditis (16 and 10\%, respectively). Myasthenia gravis may progress to involve the respiratory muscles, which may partially explain the high fatality rate. The association with myocarditis and myositis may also contribute $[12,16]$, and will require additional biologic characterization to explain this co-occurrence. Many cases of myasthenia gravis also reported cardiac arrhythmias or myocardial infarction even in cases where myocarditis was not reported, suggesting that myocarditis may be more common than recognized, and highlighting the need for diagnostic assessment for myocarditis and myositis (assessing creatinine kinase and troponin I) in patients with myasthenia gravis. Notably, the myasthenia gravis associated with ICI may be distinct from the de novo syndrome, as acetylcholinesterase receptor antibodies are frequently negative, and there is a high incidence of concurrent myositis [13].

Importantly, we did not observe additional signals of neurotoxicities. One somewhat surprising negative finding was the lack of association between ICI and demyelinating disorders such as multiple sclerosis. Multiple sclerosis is characterized by demyelination, axonal degeneration, and intense inflammation in affected lesions. This inflammation is characterized by $\mathrm{T}$ cell, $\mathrm{B}$ cell, and macrophage involvement, and improves with corticosteroids and interferon- $\beta$ (and would thus be predicted to be triggered by ICI as with numerous other inflammatory disorders) [23]. Interestingly, demyelinating disorders actually appeared to be negatively correlated with ICI use $(0.08 \%$ of ICI reports vs. $0.47 \%$ for the full database; $\left.\mathrm{IC}_{025}:-3.07\right)$, although this study was not designed to conclusively exclude drug-AE associations. The lack of associations with other neurologic disorders was also an important finding, given evidence that suggests inflammation may play a critical role in diverse neurologic conditions such as dementia, cerebrovascular disease, amytrophic lateral sclerosis, and others [24-26]. However, long-term data will be needed to definitively rule out such associations.

There are several limitations to the study intrinsic to Vigibase. First, adverse event reporting is voluntary, and comes from heterogeneous sources (e.g. physicians, pharmacists, other clinicians), thus raising the possibility of incomplete information. However, > 130 countries contribute to the database, thus ensuring an unparalleled global assessment in diverse clinical settings. Second, detailed clinical information and diagnostic criteria is unavailable, thus limiting our assessment to the treating clinician's reporting (e.g. we do not have electromyogram or cerebrospinal fluid data to confirm reported diagnoses). This may introduce bias in several directions, including either under-reporting (e.g. only reporting the most severe or obvious cases), or over-reporting (reporting cases without a firmly established diagnosis). This study does, however, complement other detailed descriptions of these clinical syndromes [6-11]. Third, we are unable to definitively determine the incidence of each event using Vigibase, although other studies have reported incidence of neurologic toxicities in the range of $1-5 \%$ [7, 8]. Fourth, changing awareness of toxicities over time could influence reporting. For example, our group and others published high-profile papers showing a link between myocarditis, myositis, and myasthenia gravis [12, 27]. These and other publications could have prompted improved awareness and potentially increased reporting over time. Finally, comparisons with anti-CTLA-4 and anti-PD-1/PD-L1 monotherapy could be confounded by disease-specific (rather than treatment specific) factors. Melanoma patients largely comprise the anti-CTLA-4 group, and may have distinct demographic and toxicity proclivities compared with the more pan-tumor population treated with anti-PD-1/PD-L1. As with other pharmacovigilance studies, this study allows for signal detection in a large population, which will need prospective and long-term validation of findings.

\section{Conclusions}

In conclusion, several categories of neurologic toxicities were strongly associated with ICI use relating to CNS inflammation (encephalitis/myelitis, meningitis, and CNS vasculitis) or peripheral neuromuscular autoimmune disorders (Guillain-Barre and myasthenia gravis). Of equal importance, no signals of other neurologic toxicities were observed, including demyelinating disorders or cerebrovascular disease. Clinicians should be aware of, and monitor for these potentially severe irAEs in patients receiving ICI therapy. 


\section{Additional file}

Additional file 1: Table S1. Neuro-psychiatric adverse events grouping as a function of Medical Dictionary for Regulatory Activities (MedDRA) Classification Version 21.0. Table S2. Example of calculating reporting odds ratio (ROR) in Vigibase. Table S3. Clinical characteristics of patients with ICl-associated cerebral vasculitis collected from VigiBase. Table S4. Clinical characteristics of patients with ICl-associated myasthenia gravis collected from VigiBase. Table S5. Clinical characteristics of patients with ICl-associated encephalitis/myelitis collected from VigiBase. Table S6. Clinical characteristics of patients with ICl-associated Guillain-Barre Syndrome collected from VigiBase. Table S7. Clinical characteristics of patients with $\mathrm{ICl}$-associated non-infectious meningitis collected from VigiBase. (DOCX 38 kb)

\section{Acknowledgements}

The supplied data from VigiBase come from a variety of sources. The likelihood of a causal relationship is not the same in all reports. The information does not represent the opinion of the World Health Organization (WHO)

\section{Funding}

This study was supported by The Cancer ITMO of the French National Alliance for Life and Health Sciences (AVIESAN): "Plan Cancer 2014-2019" (JES, BLV) by NIH/NCI K23 CA204726 (DBJ), the James C. Bradford Jr. Melanoma Fund (DBJ), the Melanoma Research Foundation (DBJ) and NIH/NHLBI R56 HL141466 (JJM)

\section{Availability of data and materials}

All data is publicly available in the Vigibase platform. https://www.who-umc. org/vigibase/vigibase/

\section{Authors' contributions}

DBJ, JJM, and JES designed the study, DBJ, JES, AM, AMH, and HTQ performed data analysis, all other authors contributed to writing the manuscript and read and approved the manuscript. All authors read and approved the final manuscript.

\section{Authors' information}

Not applicable

\section{Ethics approval and consent to participate}

All clinical data was de-identified and in a publicly available database (Vigibase).

\section{Consent for publication}

Not applicable.

\section{Competing interests}

DBJ serves on advisory boards for Array Biopharma, BMS, Genoptix, Incyte, and Merck, and receives research funding from BMS and Incyte. JJM serves on advisory boards for Pfizer, Novartis, BMS, Takeda, Myokardia, Regeneron, and Decipher, and receives research funding from BMS and Pfizer.

\section{Publisher's Note}

Springer Nature remains neutral with regard to jurisdictional claims in published maps and institutional affiliations.

\section{Author details}

'Department of Medicine, Vanderbilt University Medical Center, 777 PRB, 2220 Pierce Ave, Nashville, TN 37232, USA. ²Department of Pharmacology, Sorbonne Université, INSERM CIC Paris-Est, AP-HP, ICAN, Regional Pharmacovigilance Centre, Pitié-Salpêtrière Hospital, Paris, France. ${ }^{3}$ Muscle Disease Unit, Laboratory of Muscle Stem Cells and Gene Regulation, National Institute of Arthritis and Musculoskeletal and Skin Diseases, National Institutes of Health, Bethesda, MD, USA.
Received: 11 March 2019 Accepted: 13 May 2019

Published online: 22 May 2019

\section{References}

1. Yarchoan M, Hopkins A, Jaffee EM. Tumor mutational burden and response rate to PD-1 inhibition. N Engl J Med. 2017:377:2500-1.

2. Ribas A, Wolchok JD. Cancer immunotherapy using checkpoint blockade. Science. 2018;359:1350-5.

3. Postow MA, Sidlow R, Hellmann MD. Immune-related adverse events associated with immune checkpoint blockade. N Engl J Med. 2018;378:158-68.

4. Johnson DB, Chandra S, Sosman JA. Immune checkpoint inhibitor toxicity in 2018. JAMA. 2018

5. Wang DY, Salem JE, Cohen JV et al. Fatal toxic effects associated with immune checkpoint inhibitors: a systematic review and meta-analysis. JAMA Oncol. 2018.

6. Johnson DB, Saranga-Perry V, Lavin PJ, et al. Myasthenia gravis induced by Ipilimumab in patients with metastatic melanoma. J Clin Oncol. 2015;33:e122-4.

7. Larkin J, Chmielowski B, Lao CD, et al. Neurologic serious adverse events associated with Nivolumab plus Ipilimumab or Nivolumab alone in advanced melanoma, including a case series of encephalitis. Oncologist. 2017;22:709-18

8. Spain L, Walls G, Julve M, et al. Neurotoxicity from immune-checkpoint inhibition in the treatment of melanoma: a single Centre experience and review of the literature. Ann Oncol. 2017:28:377-85.

9. Garcia CA, El-Ali A, Rath TJ, et al. Neurologic immune-related adverse events associated with adjuvant ipilimumab: report of two cases. J Immunother Cancer. 2018:6:83.

10. Zimmer L, Goldinger SM, Hofmann L, et al. Neurological, respiratory, musculoskeletal, cardiac and ocular side-effects of anti-PD-1 therapy. Eur J Cancer. 2016;60:210-25.

11. Cuzzubbo S, Javeri F, Tissier M, et al. Neurological adverse events associated with immune checkpoint inhibitors: review of the literature. Eur J Cancer. 2017:73:1-8.

12. Moslehi JJ, Salem JE, Sosman JA, et al. Increased reporting of fatal immune checkpoint inhibitor-associated myocarditis. Lancet. 2018;391:933.

13. Anquetil C, Salem JE, Lebrun-Vignes B, et al. Immune checkpoint inhibitorassociated myositis. Circulation. 2018:138:743-5.

14. Wright JJ, Salem JE, Johnson DB, et al. Increased reporting of immune checkpoint inhibitor-associated diabetes. Diabetes Care. 2018.

15. Arnaud L, Lebrun-Vignes B, Salem JE. Checkpoint inhibitor-associated immune arthritis. Ann Rheum Dis. 2018.

16. Salem JE, Manouchehri A, Moey M, et al. Cardiovascular toxicities associated with immune checkpoint inhibitors: an observational, retrospective, pharmacovigilance study. Lancet Oncol. 2018.

17. Lindquist M. VigiBase, the WHO global ICSR database system: basic facts. Drug Inf J. 2008:42:409-19.

18. Bate $A$, Lindquist $M$, Edwards $\mathbb{R}$, et al. A Bayesian neural network method for adverse drug reaction signal generation. Eur J Clin Pharmacol. 1998:54:315-21.

19. Noren GN, Hopstadius J, Bate A. Shrinkage observed-to-expected ratios for robust and transparent large-scale pattern discovery. Stat Methods Med Res. 2013;22:57-69.

20. De Bruin ML, Pettersson M, Meyboom RH, et al. Anti-HERG activity and the risk of drug-induced arrhythmias and sudden death. Eur Heart J. 2005;26: 590-7.

21. Rothman KJ, Lanes S, Sacks ST. The reporting odds ratio and its advantages over the proportional reporting ratio. Pharmacoepidemiol Drug Saf. 2004;13: 519-23.

22. Grouthier V, Lebrun-Vignes B, Glazer AM, et al. Increased long QT and torsade de pointes reporting on tamoxifen compared with aromatase inhibitors. Heart. 2018

23. Nylander A, Hafler DA. Multiple sclerosis. J Clin Invest. 2012:122:1180-8.

24. McCauley ME, Baloh RH. Inflammation in ALS/FTD pathogenesis. Acta Neuropathol. 2018.

25. Mishra A, Brinton RD. Inflammation: bridging age, menopause and APOEepsilon4 genotype to Alzheimer's disease. Front Aging Neurosci. 2018; 10:312

26. Hu X, Leak RK, Thomson AW, et al. Promises and limitations of immune cellbased therapies in neurological disorders. Nat Rev Neurol. 2018;14:559-68.

27. Johnson DB, Balko JM, Compton ML, et al. Fulminant myocarditis with combination immune checkpoint blockade. N Engl J Med. 2016:375:1749-55. 